\title{
Non-Hispanic Black Americans' Diminished Protective Effects of Educational Attainment and Employment against Cardiometabolic Diseases: NHANES 1999-2016
}

\begin{abstract}
Zare $\mathrm{H}^{1,2 *}$ and Assari $\mathbf{S}^{3,4,5}$
${ }^{1}$ Department of Health Policy and Management, J ohns Hopkins Bloomberg School of Public Health, USA

2University of Maryland Global Campus, Health Services

Management, USA

${ }^{3}$ Marginalization-Related Diminished Returns (MDRs)

Research Center, Charles R Drew University of Medicine and Science, USA

${ }^{4}$ Department of Family Medicine, Charles R Drew University of Medicine and Science, USA

${ }^{5}$ Department of Urban Public Health, Charles R Drew University of Medicine and Science, USA

*Correspondling author: Hossein Zare, Department of Health Policy and Management, J ohns Hopkins Center for Health Disparities Solutions, J ohns Hopkins Bloomberg School of Public Health, University of Maryland Global Campus, Health Services Management, 624 North Broadway, Hampton House, Room \#337, Baltimore, Maryland, 21205, USA; Email: hzare1@jhu. edu
\end{abstract}

Received: August 26, 2021; Accepted: September 27, 2021; Published: October 04, 2021

\begin{abstract}
Background: While Socioeconomic Status (SES) indicators such as educational attainment and employment are among the major drivers of health and illness, the health returns of SES indicators may differ across racial groups. Built on the Marginalization-Related Diminished Returns framework (MDRs) that refers to weaker health effects of SES indicators for marginalized and minoritized groups than non-Hispanic White people, we conducted this study with two aims: First, to test the association between educational attainment and employment with Cardio Metabolic Diseases (CMDs), and second, to test racial variations in these associations.
\end{abstract}

Methods: This cross-sectional study used the National Health and Nutrition Examination Survey (NHANES 1999-2016) data. Participants included 29,230 adults who were either non-Hispanic White or non-Hispanic Black. We measured the following: race, demographic factors (age and sex, and marital status), SES (educational attainment and employment), behaviors (smoking, drinking, and exercise), health insurance, and CMDs (diabetes, stroke, hypertension, and congestive heart failure). Weighted Poisson regression models were used in Stata to adjust for the complex sample design of the NHANES. Models without and with interactions were performed in the pooled sample. We also ran racestratified models.

Results: Overall, high educational attainment and employment showed inverse associations with some CMDs. As documented by statistical interactions between race and our SES indicators, we observed weaker inverse associations between educational attainment and employment with some CMDs. Racestratified models also confirmed our main analysis; however, the results varied across CMD conditions.

Conclusion: We observed that SES indicators such as educational attainment and employment have differential associations for racial groups. Compared to non-Hispanic White people, non-Hispanic Black people remain at CMDs risk across the full SES spectrum. This finding is in line with the MDRs framework and may be due to the structural racism, social stratification, and marginalization of non-Hispanic Black Americans.

Keywords: Cardiometabolic disease; Diabetes; Stroke; Hypertension; Congestive heart failure; Education; Employment; Socioeconomic status; Population groups

\section{Background}

As shown and discussed by Marmot [1,2], Hayward [35], Link and Phelan [6], Ross and Miroswky [7-9], and others [10], Socioeconomic Status (SES) indicators such as educational attainment and employment are among the primary drivers of health, including but not limited to Cardio Metabolic Diseases (CMDs) such as diabetes, hypertension, stroke, and heart disease [11]. However, a growing body of research by Kaufman [12], Braveman [13], Shapiro [14,15], Williams [16,17], Ceci [18], and Navarro [19-21] has shown that SES indicators may not be comparable across racial groups; thus, the health effects of SES indicators are not equal across various social groups. To describe this phenomenon, Kaufman referred to a poor overlap between SES of racial groups as well as residual confounding of race due to unmeasured SES indicators [12]. Navarro mentions that "race and SES"-not "race or SES"-influence health disparities, which refers to the complex interplays between race and SES [19-21]. Ceci highlighted the differences between the Haves and the HaveNots in their capacity to uptake SES indicators [18]. His work argues that when resources become available, Have-Nots may be at a relative disadvantage for turning those resources into outcomes [18]. Assari recently described this phenomenon as a Marginalization-Related Diminished Returns (MDRs) phenomenon [22,23].

The MDRs phenomenon refers to weaker economic and health effects of SES indicators, particularly educational attainment and
Austin J Public Health Epidemiol - Volume 8 Issue 4 - 2021

ISSN : 2381-9014 | www.austinpublishing group.com

Zare et al. @ All rights are reserved
Citation: Zare $\mathrm{H}$ and Assari S. Non-Hispanic Black Americans' Diminished Protective Effects of Educational Attainment and Employment against Cardiometabolic Diseases: NHANES 1999-2016. Austin J Public Health Epidemiol. 2021; 8(4): 1109. 
employment, for marginalized communities, particularly racial minorities, than for US-born heterosexual non-Hispanic Whites [22,23]. These MDRs are also reported by Ferarro [24], Thorpe [25-27], Hudson [28-30], and others [31]. These studies have all documented weaker effects of SES on health for non-Hispanic Blacks than for non-Hispanic Whites. While other racial and ethnic minorities may also show some similar patterns, these MDRs are most robust for comparing non-Hispanic Blacks than for non-Hispanic Whites [22,23].

While these MDRs hold across SES indicators and health outcomes, they are best described for parental education, education, and income on mortality, self-rated health, and substance use. Less is known about the MDRs of other education and employment on Cardiometabolic Diseases (CMDs). This is important because these MDRs may be more robust for more distant (e.g., education) than proximal social determinants (e.g., employment). This is probably because more social processes can hinder the effects of educational attainment than employment on health [32]. In other terms, by the time individuals have secured employment, they have probably overcome some of the societal injustices. However, educational attainment may not result in the same employment for nonHispanic Black and non-Hispanic White people because of labor market discrimination [33]. As such, we expect stronger MDRs for educational attainment than for employment. Besides, we expect that some of the MDRs of education to be due to differential employment opportunities, thus controlling for employment may reduce the significance of MDRs due to educational attainment [32].

MDRs framework [22,23] can be regarded as a paradigm shift in health disparities research. While these MDRs are not unknown $[22,23]$ and well-established for education of non-Hispanic Blacks $[34,35]$, they are different from most of the existing literature that has traditionally focused on the role of poverty and low SES as the mechanism for racial health inequalities. Moreover, these MDRs are a paradigm shift because they: (a) seek how economic and health effects of available SES indicators vary across non-Hispanic Whites and non-Blacks, (b) explore racial disparities across the full SES spectrum and allow SES returns to vary by race, (c) use a moderated-mediation rather than a mediation model, (d) test non-linear and non-additive effects of race and SES, which are more realistic than universal average effects, and (e) explain why the racial health gap may widen rather than narrow as SES increases [22,23].

\section{Aims}

In response to the gap in the literature, we conducted a secondary multilevel analysis of the National Health and Nutrition Examination Survey (NHANES) to determine the associations between education and employment and CMDs by race. First, we hypothesize inverse associations between educational attainment and employment with CMDs. Second, built on the MDRs framework, we hypothesize that the inverse associations between educational attainment and employment with CMDs would be weaker for non-Hispanic Black than for non-Hispanic White adults. As a result, we expect a high prevalence of CMDs in non-Hispanic Blacks across educational attainment and employment levels. This will be in contrast to nonHispanic White people for whom the prevalence of CMDs would be low in highly educated and employed individuals.

\section{Materials and Methods}

We used the National Health and Nutrition Examination Survey (NHANES) data between 1999-2016 [36]. The NHANES is a crosssectional survey that provides nationally representative health and nutritional status estimates for the US population. The response rate for this data between 1999-2016 reported 73.2\% [37,38]. For this analysis, we included 29,230 individuals who were 20 years old and older. From this number, 31\% were non-Hispanic Black, and $69 \%$ were non-Hispanic White.

\section{Outcome variable}

We used five outcomes. The first four outcome variables included stroke, hypertension, diabetes, and Congestive Heart Failure (CHF). The last outcome was the presence of any CMDs, regardless of their type. We used a dummy variable for each chronic condition if the condition had been diagnosed by a doctor or any other health professional. Following the American Heart Association Guidelines, hypertension has been defined as systolic blood pressure $\geq 140 \mathrm{mmHg}$ or diastolic blood pressure $\geq 90$; the AHA modified hypertension 2017 guidelines are the most recent [39]. But, the NHANES data was collected before 2017. Therefore, in addition to the four mentioned conditions, we created a composite measure, including any of the four conditions.

\section{Main independent variable}

The main independent variables of interest were the educational level and employment. Education was defined as a categorical variable (less than high school graduate, high school graduate, or general equivalency diploma, more than high school education or some college and above). Employment was a dummy variable $(=1$, If the individual was working at a job or business or with a job or business but not at work and $=0$, if looking for a job or not working at a job or business).

\section{Covariate}

For the demographic variables, we included age (years), sex, and marital status ( $1=$ married, $0=$ otherwise). For socioeconomic status, we included income $(\$ 0-\$ 34,999,35,000-\$ 74,999$ and $\geq 75,000)$. We also included a dummy variable: having health insurance $(1=$ yes; $0=$ no). We also controlled for health behavior, including smoking (never smoked, a former smoker or current smoker), drinking (never drink, former drinker, or current drinker), and physical activity (vigorous activity).

\section{Race}

The moderator was racial/ethnic group. This was a dichotomous variable (non-Hispanic White $=0$ and non-Hispanic Black $=1$ ).

\section{Analytic strategy}

We used descriptive analysis to compare the mean and proportional differences between non-Hispanic White and nonHispanic Black people for all four conditions. Demographics, SES, and health behaviors were evaluated using unequal variances $t$-tests and chi-square. We used the weighted modified Poisson regression analysis [40-42] to produce Prevalence Ratios (PR) and the corresponding $95 \%$ confidence intervals (CI) $[40,41]$. For the first set of analyses, we ran sets of adjusted models. To find the impact of education and employment interaction on CMDs, we ran the $2^{\text {nd }}$ set of 
analyses with two interactions between race/ethnicity and education and race/ethnicity and employment status. Finally, for the last set of analyses and because the interaction between race/ethnicity and education and race/ethnicity and employment status were significant $(\mathrm{p}<0.001)$, we stratified the analyses by race. All analyses were weighted using the NHANES individual-level sampling weights for 1999-2016 (8 waves of data) to make the estimates representative at the national level for the US civilian population [43]. We considered P-values $<0.05$ as statistically significant, and all tests were twosided. We used STATA statistical software version 15 to perform all analyses.

\section{Results}

\section{Descriptive data}

A total of 29,230 individuals entered our analysis. From all participants, $12.89 \%(\mathrm{n}=9,023)$ were non-Hispanic Black and $77.11 \%$ $(\mathrm{n}=20,207)$ were non-Hispanic White. The prevalence was diabetes (7.76\%), stroke (2.68\%), hypertension (14.13\%), CHF (2.37\%), and any CMDs (22.29\%). The mean age of the participants was about 49 years $(\mathrm{SD}=11)$. Of all the participants, $64.87 \%$ were employed and $65.11 \%$ had education more than a high school degree (Table 1).

\section{Bivariate analysis}

As Table 1 shows, non-Hispanic Black participants were younger than non-Hispanic White participants. Education, income, and employment was also higher in non-Hispanic White than nonHispanic Black participants. The prevalence of smoking and drinking were also different in non-Hispanic Black and non-Hispanic White participants. While non-Hispanic Blacks were more likely to be female than non-Hispanic Whites, non-Hispanic Whites were more likely to be married. Non-Hispanic Black individuals had a higher prevalence than White participants of any CMDs and individual

Table 1: Descriptive Analysis.

\begin{tabular}{|c|c|c|c|c|c|c|c|}
\hline & \multicolumn{2}{|c|}{ Non-Hispanic White $(n=20,207)$} & \multicolumn{2}{|c|}{ Non-Hispanic Black $(n=9,023)$} & \multicolumn{2}{|c|}{ All $(n=29,230)$} & \multirow{2}{*}{$p$-value } \\
\hline & Mean & $(\mathrm{SD})$ & Mean & $(\mathrm{SD})$ & Mean & $(\mathrm{SD})$ & \\
\hline Age (years) (Mean/SD) & 48.64 & $(11.22)$ & 44.32 & $(18.5)$ & 48.09 & (12.54) & $<0.001$ \\
\hline \multicolumn{8}{|l|}{ Chronic diseases } \\
\hline Diabetes \% & 7.76 & $(17.79)$ & 12.64 & $(38.73)$ & 8.39 & (20.68) & $<0.001$ \\
\hline Stroke $\%$ & 2.68 & $(10.73)$ & 3.38 & $(21.06)$ & 2.77 & $(12.23)$ & 0.001 \\
\hline Hypertension \% & 14.13 & $(23.16)$ & 20.19 & $(46.78)$ & 14.91 & $(26.56)$ & $<0.001$ \\
\hline $\mathrm{CHF} \%$ & 2.37 & $(10.11)$ & 2.95 & $(19.73)$ & 2.44 & $(11.51)$ & 0.013 \\
\hline Any of above \% & 22.29 & $(27.67)$ & 30.62 & $(53.71)$ & 23.36 & (31.55) & $<0.001$ \\
\hline Female \% & 50.64 & $(33.24)$ & 53.15 & $(58.15)$ & 50.96 & (37.28) & $<0.001$ \\
\hline Married \% & 66.94 & $(31.27)$ & 45.07 & $(57.98)$ & 64.12 & $(35.77)$ & $<0.001$ \\
\hline \multicolumn{8}{|l|}{ Education \% } \\
\hline High school graduate/GED & 23.9 & $(28.35)$ & 25.46 & $(50.76)$ & 24.11 & $(31.9)$ & \\
\hline More than high school & 65.11 & (31.69) & 51.5 & $(58.24)$ & 63.35 & (35.93) & \\
\hline \multicolumn{8}{|l|}{ Income \% } \\
\hline$\$ 0-\$ 34,999$ & 26.79 & $(29.44)$ & 46.53 & $(58.13)$ & 29.33 & (33.95) & $<0.001$ \\
\hline $35,000-\$ 74,999$ & 33.8 & $(31.45)$ & 33.23 & $(54.89)$ & 33.73 & $(35.26)$ & \\
\hline$>=75,000$ & 39.37 & (32.48) & 19.94 & $(46.56)$ & 36.86 & (35.98) & \\
\hline Missing, DK, NA & 0.04 & $(1.37)$ & 0.3 & $(6.35)$ & 0.08 & $(2.04)$ & \\
\hline Covered by health insurance \% & 87.53 & $(21.96)$ & 76.54 & (49.38) & 86.12 & $(25.79)$ & $<0.001$ \\
\hline Did not have vigorous or Moderate Activities \% & 37.79 & $(32.23)$ & 47.92 & $(58.22)$ & 39.1 & (36.39) & $<0.001$ \\
\hline \multicolumn{8}{|l|}{ Smoking \% } \\
\hline Current smoker & 21.88 & $(27.49)$ & 25.95 & $(51.09)$ & 22.41 & (31.09) & \\
\hline \multicolumn{8}{|l|}{ Drinking \% } \\
\hline Never drink & 9 & $(19.03)$ & 16.73 & $(43.49)$ & 10 & (22.37) & $<0.001$ \\
\hline Former drinker & 11.06 & $(20.85)$ & 17.5 & $(44.28)$ & 11.89 & $(24.14)$ & \\
\hline Current drinker & 79.94 & $(26.62)$ & 65.78 & $(55.29)$ & 78.12 & (30.83) & \\
\hline Have a job/business \% & 64.87 & (31.74) & 62.02 & $(56.56)$ & 64.51 & (35.68) & 0.005 \\
\hline
\end{tabular}

Notes: 1) P-value shows the unequal variances t-tests between NHW and NHB; 2) For the categorical variables (education, income, smoking, and drinking), the p-values show the chi-sq test results. 3) All values have been weighted. 
conditions, namely diabetes, stroke, hypertension, and CHF (Table $1)$.

\section{Pooled sample models}

Table 2 shows the regression models in the pooled sample. According to the models without an interaction term (Models 1), age was positively associated with CMDs overall and individual conditions. Female sex was associated with a lower prevalence of any $\mathrm{CMD}$, diabetes, and CHF conditions. Marital status was not associated with any of the outcomes. The year of the study was positively correlated with any CMDs and diabetes and inversely associated with CHF. Being employed was inversely associated with any CMDs as well as with all individual conditions, with hypertension being the only exception. Smoking status was associated with any CMDs as well as with all individual conditions. Not having vigorous physical activity was associated with higher odds of CMDs as well as with all individual conditions. Having health insurance was associated with higher diabetes and stroke and lower odds of hypertension. Drinking status was associated with diabetes and any CMDs, but not with stroke, hypertension, and CHF. High income was associated with lower odds of any CMDs as well as with all individual conditions.
Being employed was associated with lower odds of any CMDs as well as with all individual conditions, with hypertension being the only exception. Higher than high school education was associated with lower odds of any CMDs, diabetes, or CHF but not with stroke and hypertension. According to the models without an interaction term (Models 1), the inverse associations between education and diabetes and $\mathrm{CHF}$ were significantly weaker for non-Hispanic Black than for non-Hispanic White individuals. According to this model, the inverse associations between employment and hypertension and any CHFs were significantly weaker for non-Hispanic Black than for nonHispanic White individuals (Table 2).

\section{Race-stratified models}

Table 3 shows regression results specific for each race. For nonHispanic Whites, there were inverse associations between education higher than high school and diabetes, CHF, and any CMDs. For non-Hispanic Blacks, no association was found between education higher than high school and any of the outcomes. For non-Hispanic Whites, there were inverse associations between employment and all outcomes, with hypertension being the only exception. For nonHispanic Blacks, there was a positive association between employment

Table 2: Poisson regression estimates overall.

\begin{tabular}{|c|c|c|c|c|c|c|c|c|c|c|}
\hline & \multicolumn{5}{|c|}{ Models 1} & \multicolumn{5}{|c|}{ Models 2} \\
\hline & Diabetes PR/ci95 & Stroke PR/ci95 & $\begin{array}{l}\text { Hyper-tension } \\
\text { PR/ci95 }\end{array}$ & CHF PR/ci95 & All PR/ci95 & Diabetes PR/ci95 & Stroke PR/ci95 & $\begin{array}{l}\text { Hyper-tension } \\
\text { PR/ci95 }\end{array}$ & CHF PR/ci95 & All PR/ci95 \\
\hline Age (years) & $1.03^{\star \star \star}[1.03-1.04]$ & $1.04^{\star \star \star}[1.03-1.05]$ & $1.05^{\star \star \star}[1.05-1.06]$ & $1.05^{\star \star \star}[1.05-1.06]$ & $1.03^{\star \star \star}[1.03-1.04]$ & $1.03^{\star \star \star}[1.03-1.04]$ & $1.04^{\star \star \star}[1.03-1.05]$ & $1.05^{\star \star \star}[1.05-1.06]$ & $1.05^{\star \star \star}[1.05-1.06]$ & $1.03^{\star \star \star}[1.03-1.04]$ \\
\hline Female & $0.77^{\star \star \star}[0.69-0.85]$ & $1.01[0.84-1.22]$ & $0.95[0.89-1.01]$ & $0.70^{\star \star \star}[0.59-0.82]$ & $0.93^{\star \star}[0.89-0.97]$ & $0.77^{\star \star \star}[0.69-0.85]$ & $1.01[0.83-1.22]$ & $0.95[0.89-1.01]$ & $0.70^{\star \star \star}[0.59-0.83]$ & $0.93^{\star \star}[0.89-0.97]$ \\
\hline Non-Hispanic Black & $1.81^{\star \star \star}[1.66-1.96]$ & $1.33^{\star \star \star}[1.15-1.55]$ & $1.71^{\star \star \star}[1.60-1.83]$ & $1.45^{\star \star \star}[1.24-1.69]$ & $1.42^{\star \star \star}[1.37-1.48]$ & $1.58^{\star \star \star}[1.37-1.83]$ & $1.2[0.92-1.57]$ & $1.59^{\star \star \star}[1.40-1.81]$ & $1.18[0.94-1.47]$ & $1.32^{\star \star \star}[1.23-1.42]$ \\
\hline Married & $1.04[0.94-1.15]$ & $1.1[0.94-1.28]$ & $1.02[0.94-1.10]$ & $0.9[0.76-1.05]$ & $1.02[0.98-1.07]$ & $1.04[0.94-1.15]$ & $1.1[0.94-1.28]$ & $1.01[0.94-1.10]$ & $0.9[0.76-1.06]$ & $1.02[0.98-1.07]$ \\
\hline \multicolumn{11}{|l|}{$\begin{array}{l}\text { Education (Ref. If less } \\
\text { than high school) }\end{array}$} \\
\hline High school graduate/GED & $0.93[0.82-1.06]$ & $0.99[0.82-1.19]$ & $1.09[1.00-1.20]$ & $0.89[0.73-1.08]$ & $1.02[0.97-1.08]$ & $0.9[0.77-1.05]$ & $0.99[0.80-1.22]$ & $1.1[0.98-1.23]$ & $0.81[0.64-1.03]$ & $1.01[0.95-1.09]$ \\
\hline More than high school & $0.89 *[0.80-1.00]$ & $0.84[0.68-1.04]$ & $0.99[0.91-1.08]$ & $0.81^{\star}[0.67-0.99]$ & $0.95^{\star}[0.90-1.00]$ & $0.83^{\star \star}[0.72-0.96]$ & $0.79[0.62-1.02]$ & $0.99[0.90-1.10]$ & $0.74^{\star \star}[0.59-0.92]$ & $0.93^{\star}[0.87-0.99]$ \\
\hline \multicolumn{11}{|l|}{ Income (Ref. if \$0-\$34,999) } \\
\hline $35,000-\$ 74,999$ & $0.87^{\star}[0.79-0.97]$ & $0.77^{\star \star}[0.66-0.90]$ & $0.98[0.91-1.05]$ & $0.75^{\star \star}[0.62-0.92]$ & $0.93^{\star}[0.88-0.98]$ & $0.88^{\star}[0.79-0.97]$ & $0.77^{\star \star}[0.66-0.91]$ & 0.98 [0.90-1.05] & $0.76^{\star \star}[0.62-0.92]$ & $0.93^{\star}[0.88-0.98]$ \\
\hline$>=75,000$ & $0.72^{\star \star \star}[0.63-0.83]$ & $0.52^{\star \star \star}[0.40-0.68]$ & $0.86^{\star \star}[0.77-0.95]$ & $0.42^{\star \star \star}[0.31-0.58]$ & $0.80^{\star \star \star}[0.75-0.86]$ & $0.73^{\star \star \star}[0.64-0.83]$ & $0.52^{\star \star \star}[0.40-0.69]$ & $0.86^{\star \star}[0.78-0.96]$ & $0.42^{\star \star \star}[0.31-0.58]$ & $0.81^{\star \star \star}[0.75-0.87]$ \\
\hline Missing, DK, NA & $0.26[0.06-1.07]$ & $0.63[0.08-4.76]$ & $1.13[0.48-2.67]$ & $1.63[0.42-6.27]$ & $0.86[0.50-1.47]$ & $0.26[0.06-1.09]$ & $0.62[0.08-4.69]$ & $1.16[0.49-2.75]$ & $1.68[0.43-6.52]$ & $0.87[0.50-1.52]$ \\
\hline Covered by health insurance & $1.48^{\star \star \star}[1.25-1.76]$ & $1.39 *[1.01-1.91]$ & $0.80^{\star \star \star}[0.70-0.90]$ & $1.24[0.85-1.80]$ & 1 [0.91-1.09] & $1.48^{\star \star \star}[1.24-1.75]$ & $1.39 *[1.01-1.91]$ & $0.80^{\star \star \star}[0.71-0.91]$ & $1.23[0.85-1.79]$ & 1 [0.91-1.09] \\
\hline $\begin{array}{l}\text { Did not have vigorous or } \\
\text { Moderate Activities }\end{array}$ & $1.42^{\star \star \star}[1.28-1.56]$ & $1.48^{\star \star \star}[1.24-1.77]$ & $1.09^{\star}[1.02-1.17]$ & $1.40^{\star \star \star}[1.16-1.69]$ & $1.12^{\star \star \star}[1.08-1.17]$ & $1.41^{\star \star \star}[1.28-1.56]$ & $1.48^{\star \star \star}[1.24-1.76]$ & $1.09 \star[1.02-1.17]$ & $1.40^{\star \star \star}[1.16-1.69]$ & $1.12^{\star \star \star}[1.08-1.17]$ \\
\hline \multicolumn{11}{|l|}{$\begin{array}{l}\text { Smoking (Ref. never } \\
\text { smoked) }\end{array}$} \\
\hline Former smoker & $1.21^{\star \star \star}[1.09-1.34]$ & $1.28^{\star \star}[1.09-1.52]$ & $0.99[0.91-1.07]$ & $1.49^{\star \star \star}[1.20-1.84]$ & $1.07^{\star \star}[1.02-1.12]$ & $1.21^{\star \star \star}[1.09-1.33]$ & $1.28^{\star \star}[1.08-1.52]$ & $0.99[0.91-1.07]$ & $1.48^{* \star \star}[1.19-1.83]$ & $1.07^{\star \star}[1.02-1.12]$ \\
\hline Current smoker & $0.97[0.84-1.12]$ & $1.77^{\star \star \star}[1.42-2.22]$ & $1.11 *[1.00-1.22]$ & $1.63^{\star \star \star}[1.25-2.12]$ & $1.12^{\star \star}[1.04-1.20]$ & $0.97[0.84-1.12]$ & $1.77^{\star \star \star}[1.42-2.21]$ & $1.11^{\star}[1.01-1.23]$ & $1.62^{\star \star \star}[1.24-2.11]$ & $1.12^{\star \star}[1.04-1.21]$ \\
\hline \multicolumn{11}{|l|}{$\begin{array}{l}\text { Drinking (Ref. never } \\
\text { drunk) }\end{array}$} \\
\hline Former drinker & $1.04[0.89-1.20]$ & $0.88[0.67-1.14]$ & $1.04[0.94-1.14]$ & $1.25[0.95-1.64]$ & $1.03[0.97-1.09]$ & $1.03[0.89-1.20]$ & $0.87[0.67-1.14]$ & 1.04 [0.94-1.14] & $1.25[0.95-1.64]$ & 1.03 [0.97-1.09] \\
\hline Current drinker & $0.75^{\star \star \star}[0.65-0.86]$ & 0.81 [0.63-1.03] & $0.94[0.85-1.03]$ & $0.96[0.75-1.23]$ & $0.90^{\star \star \star}[0.85-0.96]$ & $0.75^{\star \star \star}[0.65-0.86]$ & $0.81[0.63-1.03]$ & $0.94[0.85-1.03]$ & $0.96[0.75-1.23]$ & $0.90^{\star \star \star}[0.85-0.96]$ \\
\hline Have a job/business & $0.81^{\star \star}[0.70-0.92]$ & $0.36^{\star \star \star}[0.28-0.46]$ & $1.04[0.95-1.13]$ & $0.50^{\star \star \star}[0.38-0.67]$ & $0.87^{\star \star \star}[0.82-0.93]$ & $0.81^{\star}[0.69-0.96]$ & $0.35^{\star \star \star}[0.27-0.46]$ & 1 [0.90-1.10] & $0.54^{\star \star \star}[0.39-0.74]$ & $0.84^{\star \star \star}[0.78-0.91]$ \\
\hline \multicolumn{11}{|l|}{ Race $x$ Education } \\
\hline $\begin{array}{l}\text { non-Hispanic Black with } \\
\text { high school graduate/GED }\end{array}$ & & & & & & $1.11[0.92-1.35]$ & $0.93[0.64-1.35]$ & $0.95[0.81-1.12]$ & $1.53^{\star}[1.05-2.24]$ & 1 [0.91-1.11] \\
\hline $\begin{array}{l}\text { non-Hispanic Black with } \\
\text { more than high school }\end{array}$ & & & & & & $1.33^{\star \star}[1.10-1.62]$ & $1.37[0.94-2.00]$ & 0.97 [0.84-1.12] & $1.64^{\star \star}[1.15-2.33]$ & $1.07[0.98-1.17]$ \\
\hline \multicolumn{11}{|l|}{ Race x Employment } \\
\hline $\begin{array}{l}\text { non-Hispanic Black who has } \\
\text { business }\end{array}$ & & & & & & $0.94[0.79-1.12]$ & $1.04[0.70-1.54]$ & $1.24^{\star \star \star}[1.10-1.39]$ & $0.67[0.43-1.03]$ & $1.17^{\star \star \star}[1.07-1.28]$ \\
\hline $\mathbf{N}$ & 22,762 & 23,224 & 22,550 & 23,197 & 23,252 & 22,762 & 23,224 & 22,550 & 23,197 & 23,252 \\
\hline
\end{tabular}

${ }^{*} \mathrm{p}<0.05,{ }^{* *} \mathrm{p}<0.01,{ }^{* * *} \mathrm{p}<0.001$. We controlled models for year. 
Table 3: Poisson regression estimators by race.

\begin{tabular}{|c|c|c|c|c|c|c|c|c|c|c|}
\hline & \multicolumn{5}{|c|}{ Non-Hispanic White } & \multicolumn{5}{|c|}{ Non-Hispanic Black } \\
\hline & $\begin{array}{l}\text { Diabetes PRI } \\
\text { Cl95 }\end{array}$ & Stroke PR/CI95 & $\begin{array}{l}\text { Hypertension } \\
\text { PR/CI95 }\end{array}$ & CHF PR/CI95 & All PR/Cl95 & Diabetes PR/CI95 & Stroke PR/CI95 & $\begin{array}{l}\text { Hypertension } \\
\text { PR/Cl95 }\end{array}$ & CHF PR/CI95 & All PR/CI95 \\
\hline Age (years) & $1.03^{\star \star \star}[1.03-1.03]$ & $1.04^{\star \star \star}[1.03-1.05]$ & $1.05^{\star \star \star}[1.05-1.06]$ & $1.06^{* \star \star}[1.05-1.07]$ & $1.03^{\star \star \star}[1.03-1.04]$ & $1.04^{\star \star \star}[1.03-1.04]$ & $1.04^{\star \star \star}[1.03-1.05]$ & $1.05^{\star \star \star}[1.04-1.05]$ & $1.04^{\star \star \star}[1.03-1.05]$ & $1.03^{\star \star \star}[1.03-1.03]$ \\
\hline Female & $0.73^{\star \star \star}[0.64-0.82]$ & $1[0.80-1.25]$ & $0.95[0.88-1.03]$ & $0.66^{\star \star \star}[0.54-0.80]$ & $0.92^{\star \star}[0.88-0.97]$ & $0.97[0.86-1.11]$ & $1.04[0.81-1.32]$ & $0.94[0.85-1.03]$ & $0.95[0.72-1.26]$ & $0.98[0.91-1.04]$ \\
\hline Married & $1.02[0.90-1.16]$ & $1.1[0.91-1.32]$ & 1 [0.91-1.10] & $0.93[0.77-1.11]$ & $1.02[0.97-1.07]$ & $1.14^{*}[1.01-1.28]$ & $1.12[0.87-1.45]$ & $1.07[0.96-1.19]$ & $0.8[0.61-1.03]$ & 1.05 [0.98-1.12] \\
\hline \multicolumn{11}{|l|}{$\begin{array}{l}\text { Education (Ref. If less } \\
\text { than high school) }\end{array}$} \\
\hline High school graduate/GED & $0.91[0.78-1.06]$ & 0.99 [0.80-1.23] & $1.1[0.99-1.23]$ & $0.83[0.65-1.05]$ & $1.02[0.95-1.09]$ & 1 [0.88-1.15] & $0.9[0.66-1.22]$ & $1.02[0.90-1.15]$ & $1.18[0.87-1.60]$ & $0.99[0.92-1.07]$ \\
\hline More than high school & $0.85^{\star}[0.74-0.98]$ & $0.8[0.62-1.03]$ & 1 [0.90-1.10] & $0.76^{\star}[0.61-0.95]$ & $0.94^{\star}[0.88-1.00]$ & $1.05[0.91-1.22]$ & $1[0.75-1.34]$ & $0.94[0.84-1.06]$ & $1.02[0.76-1.39]$ & $0.96[0.90-1.02]$ \\
\hline \multicolumn{11}{|l|}{ Income (Ref. if $\$ 0-\$ 34,999)$} \\
\hline $35,000-\$ 74,999$ & $0.84^{\star *}[0.74-0.96]$ & $0.78^{\star \star}[0.65-0.93]$ & 1 [0.91-1.09] & $0.71^{\star \star}[0.57-0.89]$ & $0.93^{\star}[0.88-1.00]$ & 1 [0.89-1.13] & $0.75[0.55-1.03]$ & $0.9[0.80-1.03]$ & $1.03[0.77-1.38]$ & $0.93[0.86-1.00]$ \\
\hline$>=75,000$ & $0.70^{\star \star \star}[0.59-0.83]$ & $0.51^{\star \star \star}[0.37-0.69]$ & $0.89[0.79-1.00]$ & $0.38^{\star \star \star}[0.26-0.55]$ & $0.80^{\star \star \star}[0.74-0.88]$ & $0.82^{\star}[0.68-0.99]$ & $0.68[0.47-1.00]$ & $0.80^{\star}[0.66-0.96]$ & $0.88[0.58-1.33]$ & $0.84^{\star \star \star}[0.76-0.93]$ \\
\hline $\begin{array}{l}\text { Covered by health } \\
\text { insurance }\end{array}$ & $1.54^{\star \star \star}[1.20-1.96]$ & 1.44 [0.95-2.18] & $0.84^{\star}[0.71-0.99]$ & $1.28[0.78-2.10]$ & $1.04[0.91-1.18]$ & $1.31^{\star \star}[1.09-1.58]$ & $1.26[0.88-1.80]$ & $0.74^{\star \star \star}[0.67-0.83]$ & $1.21[0.76-1.94]$ & $0.94[0.86-1.02]$ \\
\hline $\begin{array}{l}\text { Did not have vigorous or } \\
\text { Moderate Activities }\end{array}$ & $1.47^{\star \star \star}[1.31-1.66]$ & $1.48^{\star \star \star}[1.21-1.81]$ & $1.11^{\star}[1.02-1.21]$ & $1.37^{\star \star}[1.10-1.70]$ & $1.14^{\star \star \star}[1.08-1.20]$ & $1.16^{\star}[1.03-1.32]$ & $1.44^{\star \star}[1.12-1.86]$ & $1.02[0.93-1.12]$ & $1.47^{\star \star}[1.12-1.93]$ & $1.06[1.00-1.12]$ \\
\hline \multicolumn{11}{|l|}{$\begin{array}{l}\text { Smoking (Ref. never } \\
\text { smoked) }\end{array}$} \\
\hline Former smoker & $1.20^{\star \star}[1.07-1.35]$ & $1.26^{\star}[1.04-1.53]$ & $1.01[0.92-1.11]$ & $1.54^{\star \star \star}[1.21-1.95]$ & $1.08^{\star \star}[1.02-1.14]$ & $1.23^{\star \star}[1.06-1.42]$ & $1.48^{\star \star}[1.12-1.95]$ & $0.9[0.79-1.03]$ & $1.23[0.85-1.78]$ & $1.03[0.95-1.12]$ \\
\hline Current smoker & $0.97[0.81-1.17]$ & $1.86^{\star \star \star}[1.43-2.41]$ & $1.11[0.98-1.26]$ & $1.78^{\star \star \star}[1.29-2.47]$ & $1.13^{* \star}[1.03-1.24]$ & $0.95[0.80-1.12]$ & $1.52^{\star \star}[1.15-1.99]$ & $1.11[0.97-1.28]$ & $1.28[0.88-1.86]$ & $1.1[1.00-1.21]$ \\
\hline \multicolumn{11}{|l|}{$\begin{array}{l}\text { Drinking (Ref. never } \\
\text { drunk) }\end{array}$} \\
\hline Former drinker & $1.04[0.86-1.27]$ & $0.77[0.57-1.06]$ & $1.04[0.92-1.17]$ & $1.40^{\star}[1.02-1.92]$ & $1.02[0.95-1.10]$ & $0.99[0.84-1.17]$ & $1.45^{\star}[1.00-2.10]$ & $1.03[0.92-1.17]$ & 0.84 [0.54-1.31] & $1.04[0.97-1.12]$ \\
\hline Current drinker & $0.73^{\star \star \star}[0.61-0.88]$ & $0.77[0.58-1.02]$ & $0.91[0.81-1.02]$ & 1.02 [0.75-1.39] & $0.89^{\star \star \star}[0.83-0.95]$ & $0.84^{\star}[0.71-0.99]$ & $1.06[0.73-1.55]$ & $1.06[0.94-1.21]$ & $0.89[0.62-1.27]$ & $0.99[0.92-1.07]$ \\
\hline Employed & $0.80^{\star}[0.67-0.95]$ & $0.36^{\star \star \star}[0.27-0.48]$ & $1.02[0.92-1.13]$ & $0.59^{\star \star}[0.43-0.83]$ & $0.86^{\star \star \star}[0.80-0.93]$ & $0.78^{\star \star \star}[0.68-0.90]$ & $0.34^{\star \star \star}[0.24-0.49]$ & $1.15^{\star \star}[1.04-1.28]$ & $0.25^{\star \star \star}[0.17-0.37]$ & $0.93[0.85-1.01]$ \\
\hline $\mathbf{N}$ & 15,862 & 16,165 & 15,757 & 16,148 & 16,190 & 6,900 & 7,059 & 6,793 & 7,049 & 7,062 \\
\hline
\end{tabular}

${ }^{*} p<0.05,{ }^{* *} p<0.01,{ }^{* * *} p<0.001$. We controlled models for year.

and hypertension in non-Hispanic Black people. For non-Hispanic Blacks, there were inverse associations between employment and diabetes, stroke, $\mathrm{CHF}$, and any CMDs.

\section{Discussion}

Educational attainment and employment were associated with lower odds of several CMDs; however, race moderated these associations, and we observed weaker associations for non-Hispanic Black than for non-Hispanic White people. As a result, highly educated and employed non-Hispanic Black people remain at higherthan-expected CMDs risk.

These findings align with some recent observations that the effects of SES indicators, particularly education on obesity, heart disease, and hypertension are weaker for NHB than NHW. These MDRs also hold for chronic diseases [44-46], disability, hospitalization, and mortality. As a result of these MDRs, we observe premature mortality of highly educated and employed non-Hispanic Blacks.

As a result of the existing MDRs, highly educated racial/ethnic minority individuals show worse mental [47], behavioral [48,49], and physical health [17], and underutilize preventive healthcare $[50,51]$. In addition, poor mental health [52,53], high substance use $[49,54,55]$, poor sleep [56], and poor diet [57] may result in a higher risk of CMDs in highly educated non-Hispanic Black and Hispanic people [32,58].

While most research on this topic has focused on differential effects of education, at least some research was conducted that showed that employment may also be associated with higher health advantages for non-Hispanic Whites than for non-Hispanic Blacks $[59,60]$. In one study, employment showed higher protection again allcause mortality during a 25-year follow-up for non-Hispanic Whites than for non-Hispanic Blacks. While the highest life expectancy gain from employment went to highly educated non-Hispanic White men, and non-Hispanic White and non-Hispanic Black women still gained some life expectancy from their employment; non-Hispanic Black men did not show any protection against all-cause mortality from employment. In another study [48], employed non-Hispanic White people reported the lowest rate of smoking. Employed ethnic minority people, however, reported higher smoking [48]. Along the same lines, education has been shown to generate more income, wealth, and financial stability for non-Hispanic Whites than for nonHispanic Blacks [32,61-63]. In one study, income showed a larger increase over time for non-Hispanic Whites than for non-Hispanic Blacks [64].

Our diminished returns of education and employment are related. As education is more distal than employment, and due to labor market discrimination, education may generate worse employment and occupations for non-Hispanic Blacks than for nonHispanic Whites; the MDRs of education may be due in part to MDRs of employment. Previous work has also shown diminished health returns of employment [32] for substance use and life expectancy for non-Hispanic Blacks [59] and Hispanics [54,65].

A wide range of structural, social, and behavioral mechanisms may explain these MDRs. It is difficult to decompose the mechanism, 
particularly because educational attainment, employment, income, behaviors, and health are all associated, and most of these associations are racialized (weaker for non-Hispanic Black) [22,23]. We propose that highly educated non-Hispanic Blacks work in jobs with lower pay and lower occupational prestige, which are associated with higher stress and exposure to toxins [66]. Racial compositions of jobs may also be associated with discrimination for highly educated non-Hispanic Black employees [67]. As a result, highly educated and employed non-Hispanic Blacks [22,23] remain at risk of economic insecurity [61], stress [68], poor residential areas [69], and low wealth [63]. These complexities suggest that multiple, interwoven, complex social processes may explain why highly educated and employed nonHispanic Black people remain at behavioral, economic, and health risk.

More research should test whether work conditions and occupational prestige are why education and employment are associated with fewer health returns for non-Hispanic Blacks than for non-Hispanic Whites. More is known about the role of diet, exercise, sleep, and substance use. These behaviors are shown to be worse for highly educated and employed non-Hispanic Black people. What remains unknown is whether work conditions also explain these diminishing returns.

\section{Limitations}

This study had a few limitations. First, it used cross-sectional data, so the causal inference is not possible. While the association between employment and CMDs is bidirectional, and poor health can also reduce the likelihood of employment, this is less the case for education. Thus, the results should be interpreted with more caution about the directionality of an employment-health association. Another limitation is that the sample size was much more limited for non-Hispanic Black than for non-Hispanic White people. This is, however, the case in almost any national study. In addition, CMDs were self-reported and were not verified by health claims or laboratory data. Finally, this study did not include some confounders such as sexual orientation, diet, and other proxies of marginalization and determinants of CMDs. Despite these limitations, this paper makes a strong contribution by showing that while MDRs hold for both education and employment, these effects may depend on CMD type.

\section{Implications}

The results suggest that to eliminate racial CMDs inequalities, we may need policies beyond poverty elimination and address occupational situations that may equalize the health return of educational attainment and employment by race. Such policies that address social inequalities such as labor market discrimination or differential quality of education are hoped to reduce racial health disparities due to MDRs. This is important because solutions to health disparities due to low returns of educational attainment and employment for non-Hispanic Black people (i.e., MDRs) are different from those due to inadequate education, unemployment, and associated poverty. Thus, unless we develop policies that address MDRs in non-Hispanic Black people, and unless we go beyond poverty elimination to address CMD racial health inequalities, educational attainment and employment may continue to operate as 'a solution' as well as 'a source' of racial health disparities.

\section{Conclusion}

As shown here, SES indicators such as educational attainment and employment do not have similar associations with CMDs across racial groups. Highly educated and employed non-Hispanic Black people remain with some additional CMDs risk, a pattern different from their non-Hispanic White counterparts. Thus, racial disparities in CMDs sustain across the full SES spectrum. As proposed by the MDRs, racial health disparities should not be reduced to the problem of poverty, low education, or unemployment. These MDRs may reflect structural racism, social stratification, and marginalization that hinder non-Hispanic Black Americans across SES levels.

\section{Declaration}

Acknowledgement: Martin F. Blair provided the great edit to the manuscript.

Data availability statement: The data presented in this study are openly available in [National Health and Nutrition Examination Survey (NHANES) at https://www.cdc.gov/nchs/nhanes/index.htm].

Declaration of conflicting interests: The Author(s) declare(s) no conflict of interest.

Funding: Hossein Zare received funding from Johns Hopkins Bloomberg School of Public Health (Discretionary Fund) and Hopkins Center for Health Disparities Solutions (U54MD000214-6865). Shervin Assari is supported by the National Institutes of Health (NIH) grants 5S21MD000103, CA201415 02, DA035811-05, U54MD007598, U54MD008149, D084526-03, and U54CA229974.

\section{References}

1. Marmot M. Economic and social determinants of disease. Bull World Health Organ. 2001; 79: 988-989.

2. Marmot M. Social determinants of health inequalities. Lancet. 2005; 365 1099-1104.

3. Montez JK, Hummer RA, Hayward MD. Educational attainment and adult mortality in the United States: a systematic analysis of functional form. Demography. 2012; 49: 315-336.

4. Montez JK, Zajacova A, Hayward MD. Disparities in Disability by Educational Attainment Across US States. Am J Public Health. 2017; 107: 1101-1108.

5. Montez JK, Zajacova A, Hayward MD, Woolf SH, Chapman D, Beckfield J. Educational Disparities in Adult Mortality Across U.S. States: How Do They Differ, and Have They Changed Since the Mid-1980s? Demography. 2019; 56: 621-644.

6. Clouston SAP, Link BG. A Retrospective on Fundamental Cause Theory: State of the Literature and Goals for the Future. Annual Review of Sociology. 2021; 47: 131-156

7. Ross CE, Mirowsky J. Does employment affect health? J Health Soc Behav. 1995; 36: 230-243.

8. Ross CE, Mirowsky J. Refining the association between education and health: the effects of quantity, credential, and selectivity. Demography. 1999; 36: $445-460$.

9. Mirowsky J, Ross CE. Education, Health, and the Default American Lifestyle. J Health Soc Behav. 2015; 56: 297-306.

10. Needham BL, Smith JA, Zhao W, et al. Life course socioeconomic status and DNA methylation in genes related to stress reactivity and inflammation: The multi-ethnic study of atherosclerosis. Epigenetics. 2015; 10: 958-969.

11. Sodjinou R, Agueh V, Fayomi B, Delisle H. Obesity and cardio-metabolic 
risk factors in urban adults of Benin: relationship with socio-economic status, urbanisation, and lifestyle patterns. BMC public health. 2008; 8: 1-13.

12. Kaufman JS, Cooper RS, McGee DL. Socioeconomic status and health in blacks and whites: the problem of residual confounding and the resiliency of race. Epidemiology. 1997: 621-628

13. Braveman PA, Cubbin C, Egerter S, et al. Socioeconomic status in health research: one size does not fit all. JAMA. 2005; 294: 2879-2888.

14. Oliver M, Shapiro T. Black wealth/white wealth: A new perspective on racial inequality. Routledge. 2013.

15. Oliver ML, Shapiro TM. Black wealth/white wealth. New York: Routledge. 1999.

16. Williams DR, Costa MV, Odunlami AO, Mohammed SA. Moving upstream how interventions that address the social determinants of health can improve health and reduce disparities. J Public Health Manag Pract. 2008; 14: S8S17.

17. Williams DR. Race, socioeconomic status, and health the added effects of racism and discrimination. 1999.

18. Ceci SJ, Papierno PB. The rhetoric and reality of gap closing: when the" have-nots" gain but the" haves" gain even more. American Psychologist. 2005; 60: 149 .

19. Navarro V. Race or class or race and class: growing mortality differentials in the United States. Int J Health Serv. 1991; 21: 229-235.

20. Navarro V. Race or class versus race and class: mortality differentials in the United States. Lancet. 1990; 336: 1238-1240.

21. Navarro V. Race or class, or race and class. Int J Health Serv. 1989; 19 311-314.

22. Assari S. Unequal Gain of Equal Resources across Racial Groups. Int J Health Policy Manag. 2017; 7: 1-9.

23. Assari S. Health Disparities due to Diminished Return among Black Americans: Public Policy Solutions. Social Issues and Policy Review. 2018; 12: $112-145$

24. Farmer MM, Ferraro KF. Are racial disparities in health conditional on socioeconomic status? Soc Sci Med. 2005; 60: 191-204.

25. Wilson KB, Thorpe RJ, Jr., LaVeist TA. Dollar for Dollar: Racial and ethnic inequalities in health and health-related outcomes among persons with very high income. Prev Med. 2017; 96: 149-153.

26. Laveist TA, Thorpe RJ, Jr., Mance GA, Jackson J. Overcoming confounding of race with socio-economic status and segregation to explore race disparities in smoking. Addiction. 2007; 102: 65-70.

27. Bell CN, Sacks TK, Thomas Tobin CS, Thorpe RJ, Jr. Racial Non-equivalence of Socioeconomic Status and Self-rated Health among African Americans and Whites. SSM Popul Health. 2020; 10: 100561.

28. Hudson D, Sacks T, Irani K, Asher A. The Price of the Ticket: Health Costs of Upward Mobility among African Americans. Int J Environ Res Public Health. 2020; 17.

29. Hudson DL, Puterman E, Bibbins-Domingo K, Matthews KA, Adler NE. Race, life course socioeconomic position, racial discrimination, depressive symptoms and self-rated health. Soc Sci Med. 2013; 97: 7-14.

30. Hudson DL, Bullard KM, Neighbors HW, Geronimus AT, Yang J, Jackson JS. Are benefits conferred with greater socioeconomic position undermined by racial discrimination among African American men? J Mens Health. 2012 9: 127-136.

31. Spera C, Wentzel KR, Matto HC. Parental aspirations for their children's educational attainment: Relations to ethnicity, parental education, children's academic performance, and parental perceptions of school climate. Journal of youth and adolescence. 2009; 38: 1140-1152

32. Assari S. Blacks' Diminished Return of Education Attainment on Subjective Health; Mediating Effect of Income. Brain Sci. 2018; 8.

33. Bertrand M, Mullainathan S. Are Emily and Greg more employable than
Lakisha and Jamal? A field experiment on labor market discrimination. American economic review. 2004; 94: 991-1013.

34. Assari S, Caldwell $\mathrm{CH}$. High Risk of Depression in High-Income African American Boys. J Racial Ethn Health Disparities. 2018; 5: 808-819.

35. Assari S, Lankarani MM, Caldwell CH. Does Discrimination Explain High Risk of Depression among High-Income African American Men? Behav Sci (Basel). 2018; 8.

36. NHANES. National Center for Health Statistics. National Health and Nutrition Examination Survey. Hyattsville, MD: US Department of Health and Human Services, CDC, National Center for Health Statistics; Retrieved March $3^{\text {rd }}$ 2020. 2020.

37. Zipf G, Chiappa M, Porter KS, Ostchega Y, Lewis BG, Dostal J. National health and nutrition examination survey: plan and operations, 1999-2010. National Center for Health Statistics. Vital Health Stat. 2013; 1.

38. NHANES. Analytic Guidelines, 2011-2014 and 2015-2016. National Health and Nutrition Examination Survey. 2018.

39. Whelton PK, Carey RM, Aronow WS, et al. 2017 ACC/AHA/AAPA/ABC/ ACPM/AGS/APhA/ASH/ASPC/NMA/PCNA guideline for the prevention, detection, evaluation, and management of high blood pressure in adults: a report of the American College of Cardiology/American Heart Association Task Force on Clinical Practice Guidelines. Journal of the American College of Cardiology. 2018; 71: e127-e248.

40. Thorpe Jr RJ, Parker LJ, Cobb RJ, Dillard F, Bowie J. Association between discrimination and obesity in African-American men. Biodemography and social biology. 2017; 63: 253-261.

41. McNutt L-A, Wu C, Xue X, Hafner JP. Estimating the relative risk in cohort studies and clinical trials of common outcomes. American Journal of Epidemiology. 2003; 157: 940-943.

42. Zou G. A modified poisson regression approach to prospective studies with binary data. American Journal of Epidemiology. 2004; 159: 702-706.

43. CDC. Centers for Disease Control Prevention. National Health and Nutrition Examination Survey: Analytic Guidelines, 2011-2014 and 2015-2016. National Center for Health Statistics, editor Atlanta, GA. 2018.

44. Assari S, Moghani Lankarani M. Poverty Status and Childhood Asthma in White and Black Families: National Survey of Children's Health. Healthcare (Basel). 2018; 6.

45. Assari S, Caldwell CH. Family Income at Birth and Risk of Attention Deficit Hyperactivity Disorder at Age 15: Racial Differences. Children (Basel). 2019; 6.

46. Assari S. Socioeconomic Determinants of Systolic Blood Pressure; Minorities' Diminished Returns. Journal of Health Economics and Development. 2019; 1: 1-11.

47. Assari S, Lapeyrouse LM, Neighbors HW. Income and Self-Rated Mental Health: Diminished Returns for High Income Black Americans. Behav Sci (Basel). 2018; 8.

48. Assari S, Mistry R. Diminished Return of Employment on Ever Smoking Among Hispanic Whites in Los Angeles. Health Equity. 2019; 3: 138-144.

49. Assari S, Mistry R. Educational Attainment and Smoking Status in a National Sample of American Adults; Evidence for the Blacks' Diminished Return. Int J Environ Res Public Health. 2018; 15.

50. Assari S, Bazargan M. Educational Attainment Better Increases the Chance of Breast Physical Exam for Non-Hispanic than Hispanic American Women: National Health Interview Survey. Hospital Practices and Research. 2019; 4: $122-127$

51. Assari S, Hani N. Household Income and Children's Unmet Dental Care Need; Blacks' Diminished Return. Dent J (Basel). 2018; 6.

52. Assari S. High Income Protects Whites but Not African Americans against Risk of Depression. Healthcare (Basel). 2018; 6.

53. Assari S. Educational Attainment Better Protects African American Women than African American Men Against Depressive Symptoms and Psychological 
Distress. Brain Sci. 2018; 8

54. Assari S, Farokhnia M, Mistry R. Education Attainment and Alcohol Binge Drinking: Diminished Returns of Hispanics in Los Angeles. Behav Sci (Basel). 2019; 9.

55. Shervin A, Ritesh M. Diminished Return of Employment on Ever Smoking Among Hispanic Whites in Los Angeles. Health Equity. 2019; 3: 138-144.

56. Assari S. Parental Education and Children's Sleep Problems: Minorities' Diminished Returns. International Journal of Epidemiologic Research. 2021; 8: $31-39$

57. Assari S, Boyce S, Bazargan M, Caldwell CH, Mincy R. Maternal Education at Birth and Youth Breakfast Consumption at Age 15: Blacks' Diminished Returns. J Multidisciplinary Scientific Journal. 2020; 3: 313-323.

58. Assari S, Lankarani MM. Race and Urbanity Alter the Protective Effect of Education but not Income on Mortality. Front Public Health. 2016; 4: 100.

59. Assari S. Life Expectancy Gain Due to Employment Status Depends on Race, Gender, Education, and Their Intersections. J Racial Ethn Health Disparities. 2018; 5: 375-386.

60. Nie $Y$, Witten B, Schatell D, et al. Changes in employment status prior to initiation of maintenance hemodialysis in the USA from 2006 to 2015. Clin Kidney J. 2020; 13: 434-441.

61. Assari S. Parental Education Better Helps White than Black Families Escape Poverty: National Survey of Children's Health. Economies. 2018; 6: 30.
62. Assari S. Understanding America: Unequal Economic Returns of Years of Schooling in Whites and Blacks. World J Educ Res. 2020; 7: 78-92.

63. Assari S. College Graduation and Wealth Accumulation: Blacks' Diminished Returns. World J Educ Res. 2020; 7: 1-18.

64. Assari S, Preiser B, Kelly M. Education and Income Predict Future Emotional Well-Being of Whites but Not Blacks: A Ten-Year Cohort. Brain Sci. 2018; 8

65. Assari S, Bazargan M. Educational Attainment and Self-Rated Oral Health among American Older Adults: Hispanics' Diminished Returns. Dentistry Journal. 2019; 7: 97.

66. Assari S, Bazargan M. Unequal Effects of Educational Attainment on Workplace Exposure to Second-Hand Smoke by Race and Ethnicity; Minorities' Diminished Returns in the National Health Interview Survey (NHIS). J Med Res Innov. 2019; 3.

67. Assari S, Moghani Lankarani M. Workplace Racial Composition Explains High Perceived Discrimination of High Socioeconomic Status African American Men. Brain Sci. 2018; 8 .

68. Assari S, Bazargan M. Unequal Associations between Educational Attainment and Occupational Stress across Racial and Ethnic Groups. International Journal of Environmental Research and Public Health. 2019; 16: 3539.

69. Assari S, Boyce S, Caldwell CH, Bazargan M, Mincy R. Family Income and Gang Presence in the Neighborhood: Diminished Returns of Black Families. Urban Science. 2020; 4: 29. 